\title{
Pipeline direct digital frequency synthesiser using decomposition method
}

\author{
S.-I.Liu, T.-B.Yu and H.-W.Tsao
}

\begin{abstract}
A direct digital frequency synthesiser using a new decomposition method without the large sine ROM table is presented. To improve its operating frequency a pipeline structure has been utilised. It has been fabricated in a $0.6 \mu \mathrm{m}$ single-poly double-metal (SPDM) CMOS process and its core area is $0.95 \times 1.1 \mathrm{~mm}^{2}$. The maximum operating frequency is $85 \mathrm{MHz}$. For a $10 \mathrm{MHz}$ sinusoidal output, the phase noise is $-114 \mathrm{dBc} / \mathrm{Hz}$ at an offset frequency of $10 \mathrm{kHz}$. The measured SNR is $60.77 \mathrm{~dB}$ and worst case spurious is $-67.6 \mathrm{dBc}$. Its power dissipation is $80 \mathrm{~mW}$ at $80 \mathrm{MHz}$ under the $5 \mathrm{~V}$ supply.
\end{abstract}

\section{Introduction}

A frequency synthesiser is one of the important building blocks for wireless communication. There are two major methods of coherent frequency synthesis, direct and indirect [1]. The indirect methods are primarily phase-locked loop (PLL)-based. Direct digital frequency synthesiser (DDFS) methods can have many important advantages over the PLL-based methods, including smaller frequency resolution, fast and phase-continuous frequency switching and excellent tempcrature and ageing stability etc. However, DDFS methods may require larger chip area and power consumption. Most DDFSs use the sine table lookup method [2-4]. This method requires a large ROM to store the sinusoidal samples, although many improved methods [3 7] utilise the symmetrical property and trigonometric decomposition of the sine function to reduce the ROM size. In addition, some other methods such as the Taylor series approximation [8], CORDIC algorithm [9] and residuc number [10] computation methods have also been presented to implement the direct digital frequency synthesiser. The Taylor series approximation method nceds three small-size ROMs and large computing complexity for multiplication and the power dissipation will be large for high frequency sine wave synthesis. The CORDIC method can reduce the required ROM size efficiently, but the computation complexity is also large. The residue number method does not need any multiplication, but many small ROMS and a residue number system to binary conversion circuit are required. In this paper, new decomposition and pipeline methods are used to create a DDFS without large sine ROM lables. It has the following advantages: no multiplication, no large sine ROM, low computation complexity and low power dissipation.

\footnotetext{
(C) IEE, 2001

IEE Proceedings online no. 20010158

DOI: 10.1049/p-cds:20010158

Paper first received 8th October 1999 and in revised form 6 th November 2000 The authors are with the Department of Elcctrical Engineering \& Graduate Institute of Flectronics Engineering, National Taiwan Universily, Taipei, Titiwan 10617. Republic of China
}

\section{Circuit description}

A conventional DDFS consists of a phase accumulator, a sine ROM look-up table and a digital-to-analogue converter (DAC). A frequency control word is applied to the phase accumulator to produce the address word for the sine ROM table. The output of the ROM table is converted to a sinusoidal signal by a DAC. The proposed DDFS uses a new decomposition method. A sine function is decomposed into the summation of two functions: coarse function $f(x)$ and fine function $g(x)$ as shown in Fig. 1 . Such a sinusoidal decomposition method can be expressed as

$$
\sin x=f(x)+g(x)
$$

where $x$ is the output of the phase accumulator. According to the required signal-to-noise ratio $(>60 \mathrm{~dB}$ ), a 14-bit address word at the output of the phase accumulator and 10-bit outputs for the DAC are needed. For the coarse function as shown in Fig. 1. a quarter-wave sine waveform with the amplitude of 4095 is divided into eight sections with the magnitude of cvery section being the exponent of 2 (i.e. $2^{9}=512$ ). If the sine function is expressed in binary form, the first three most signilicant bits just represent the coarse function $f(x)$ and the remaining bits are the fine function $g(x)$. One of advantages for this kind of decomposition is that the coarse and fine functions are independent and the output of $\sin x$ can be obtained without extra

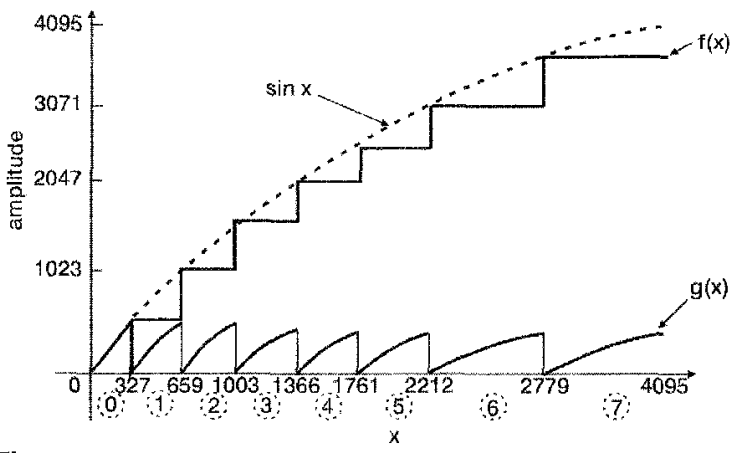

Fig. 1 Decomposition of sine function 
adders to sum $f(x)$ and $g(x)$. Moreover, according to Table 1, depending on the value of the output bits of the phase accumulator, $f(x)$ can be implemented using only some combinational logic gates. For example, if $x$ falls within the interval of $[0,326]$ (the 0th section in Table 1), the three most significant bits will be 000 . In fact, only 75 gate counts are needed to realise $f(x)$ in this DDS.

Table 1: Coarse function $f(x)$

\begin{tabular}{llll}
\hline Section & Address: $x$ & Operation & $\begin{array}{l}f(x): \text { three MSB } \\
\text { bits of } \sin x\end{array}$ \\
\hline 0 & {$[0: 326]$} & $0<=x<327$ & 000 \\
1 & {$[327: 658]$} & $327<=x<659$ & 001 \\
2 & {$[659: 1002]$} & $659<=x<1003$ & 010 \\
3 & {$[1003: 1365]$} & $1003<=x<1366$ & 011 \\
4 & {$[1366: 1760]$} & $1366<=x<1761$ & 100 \\
5 & {$[1761: 2211]$} & $1761<=x<2212$ & 101 \\
6 & {$[2212: 2778]$} & $2212<=x<2779$ & 110 \\
7 & {$[2779: 4095]$} & $2779<=x<4095$ & 111 \\
\hline
\end{tabular}

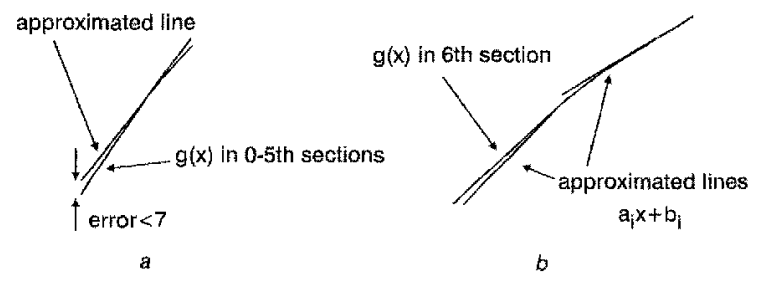

approximated lines $a_{i} x+b_{i}$

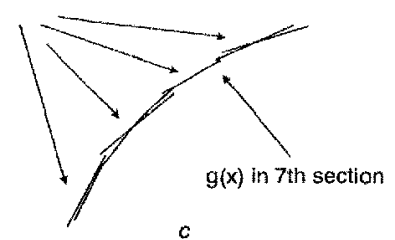

Fig.2 Approximation of fune function $g(x)$ using line segments

Table 2: Fine function, $g(x)=a_{i} \cdot x-b_{i}$ where $a_{i}$ and $b_{i}$ are constants

\begin{tabular}{llll}
\hline Section $i$ & Address: $x$ & $a_{i} \cdot x-b_{i}$ (decimal) & $a_{i} \cdot x-b_{i}$ (binary) \\
\hline 0 & {$[0: 326]$} & $1.5625 \cdot x$ & $1.1001_{2} \cdot x$ \\
1 & {$[327: 658]$} & $1.53125 \cdot x-497$ & $1.10001_{2} \cdot x-497_{10}$ \\
2 & {$[659: 1002]$} & $1.4375 \cdot x-939$ & $1.0111_{2} \cdot x-939_{10}$ \\
3 & {$[1003: 1365]$} & $1.40625 \cdot x-1409$ & $1.01101_{2} \cdot x-1409_{10}$ \\
4 & {$[1366: 1760]$} & $1.28125 \cdot x-1745$ & $1.01001_{2} \cdot x-1745_{10}$ \\
5 & {$[1761: 2211]$} & $1.125 \cdot x-1974$ & $1.001_{2} \cdot x-1974_{10}$ \\
6 & {$[2212: 2495]$} & $0.9375 \cdot x-2067$ & $0.1111_{2} \cdot x-2067_{10}$ \\
& {$[2496: 2778]$} & $0.8125 \cdot x-1747$ & $0.1101_{2} \cdot x-1747_{10}$ \\
7 & {$[2779: 3071]$} & $0.6875 \cdot x-1908$ & $0.1011_{2} \cdot x-1908_{10}$ \\
& {$[3072: 3455]$} & $0.5 \cdot x-1332$ & $0.1_{2} \cdot x-1332_{10}$ \\
& {$[3456: 3839]$} & $0.269531 \cdot x-536$ & $0.01000101_{2} \cdot x-536_{10}$ \\
& {$[3840: 4095]$} & $0.078125 \cdot x+194$ & $0.000101_{2} \cdot x+194_{10}$ \\
\hline
\end{tabular}

The approximation of the fine function $g(x)$ can be described as follows: form the oth section to the fifth section of the fine function, single straight-line segments with different slope factors $a_{i}(i=0-5)$ can be used to approximate $g(x)$ as shown in Fig. $2 a$ and the crror can be limited within $0 \sim 7$. In this way, $g(x)$ can be implemented using only adders and multiplexers without multipliers. However, if a single straight line is used to approach the ideal curve in the sixth and seventh sections, the error will be larger. Therefore, two line segments and four line segments are employed in the sixth and seventh sections as shown in Figs. $2 b$ and $c$, respectively, to keep the error below seven. The fine function, $g(x)=a_{i} \cdot x-b_{i}$ for every section $i$, has been calculated and optimised by Matlab [11] and they are listed in Table 2. Therefore the sin function $\sin x$, can be rewritten as

$$
\sin x=f(x)+g(x) \cong\left[c_{i}\right]+\left[a_{i} x-b_{i}\right]
$$

where $a_{i}$ and $b_{i}$ are the coefficients listed in Table 2 and $c_{i}$ is the output of the coarse function listed in Table 1 and $i$ is the section number. To see how operation of eqn. 2 , one may rewrite it as

$$
\sin x=f(x)+g(x) \cong\left[C_{i}\right]+\left[A_{i} x+D_{i}\right]
$$

where $A_{i}=a_{i} C_{i}=c_{i}$ and $D_{i}=1+$ the complement of $b_{i}$. Investigating the slope factors $a_{i}$ in Table 2 , one can find that the number of ' 1 's for the slope factor $a_{i}$ is no more than four. Thus the operation of $a_{i} x$ can be implemented with three adders and four shift operations but no multipliers, as shown in Fig. 3. For example, in Section 2, the fine function can be approximated as

$$
\begin{aligned}
g(x) & =(1.4375 \bullet x-939)_{10}=(1.0111 \bullet x)_{2}+3156_{10} \\
& =\left(\left(x+\frac{x}{4}\right)+\left(\frac{x}{8}+\frac{x}{16}\right)\right)_{2}+3156_{10}
\end{aligned}
$$

where the subscripts 10 and 2 denote that the numbers are represented by decimal and binary, respectively. According to eqn. 4 , the multiplication of $a_{i}$ and $x$ can be realised by applying four shift operations to $x$ and three adders. The constant can also be realised by combinational logic gates. Since the number of the slope coefficients $a_{i}$ expressed in terms of binary bits is no more than four, the fine function $g(x)$ can be realised by four adders and four shift operations. The sine function $\sin x=f(x)+g(x)$, can then be obtained without no extra adders.

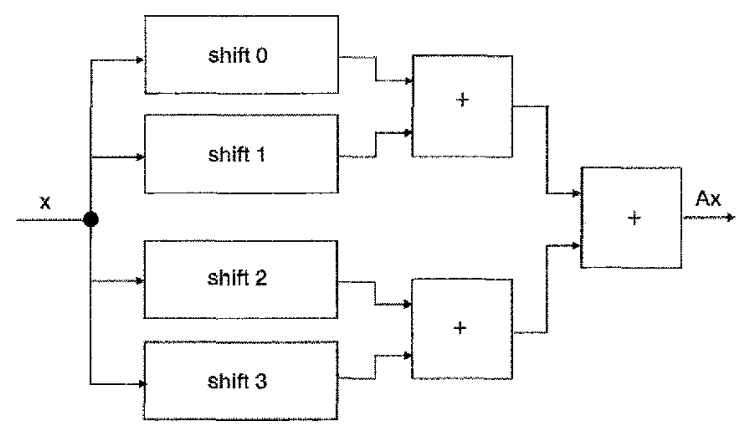

Fig. 3 Realisation of operation $a_{p} x$

Based on this decomposition method, the proposed DDFS has been designed as shown in Fig. 4. To further improve the speed of this DDFS, a pipeline structure is adopted. The whole structure is divided into four parts. The first part includes the 16-bit phase accumulator and some logic circuits to initiate the orthogonal operation. In this DDFS, logic circuits are used to detect the moment at which the sine signal goes through the positive $90^{\circ}$. Once it goes through $90^{\circ}$, a flag signal is pulled high as shown in Fig. 5. This flag signal can be used to initiate the other DDFS to provide the quadrature output. The second part realises the coarse function according to Table 1 . The third 


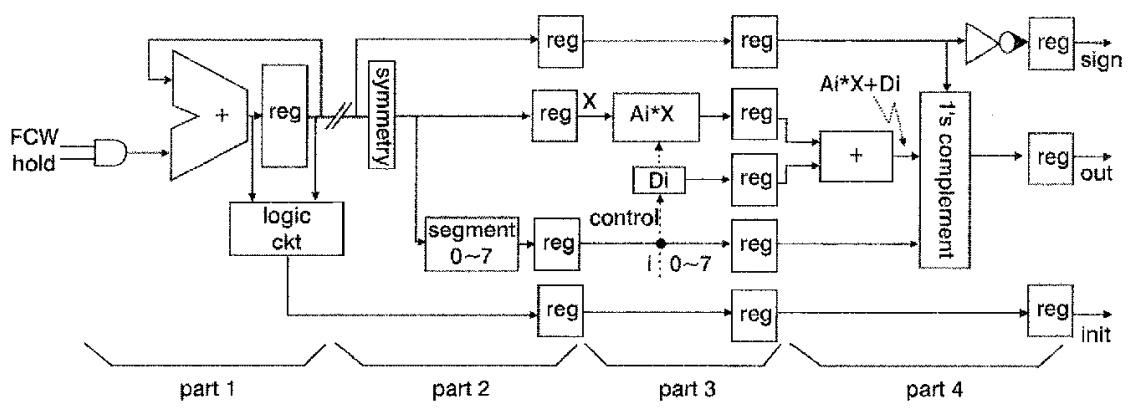

Fig. 4 Block diagram of proposed DDFS

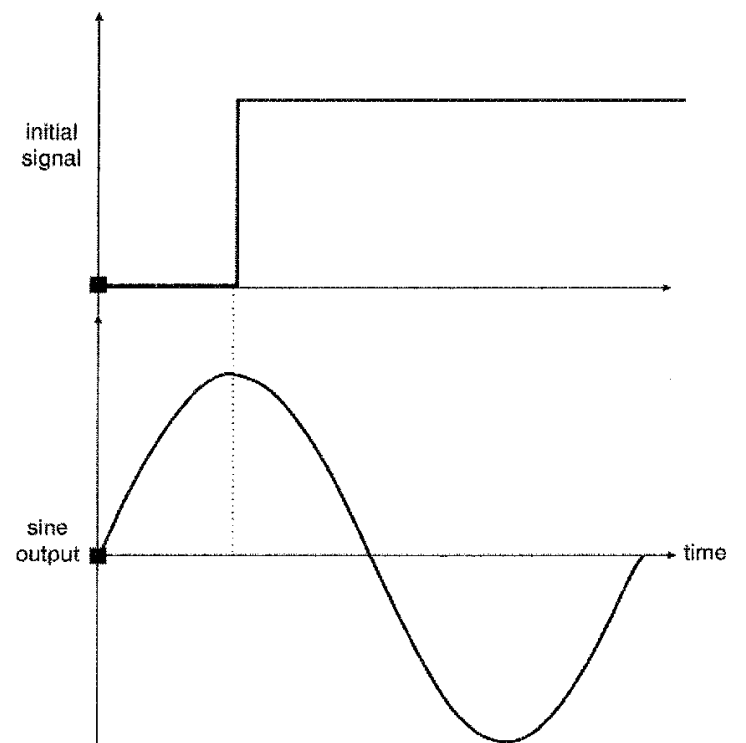

Fig.5 Timing dicgram for realising or thogonal signals

and fourth parts realise the fine function and output the digital sinusoidal signal using the l's complement adder. The 16-bit adder employed in the phase accumulator is the so-called carry select adder $[12,13]$.

To estimate the delay time in each part of the proposed DDFS, the Synopsys tool [14] is utilised. For example, the calculated delay time for the 16-bit carry select adder in the phase accumulator is about $5.3 \mathrm{~ns}$. The remaining components have also been calculated as listed in Table 3. After the post simulations, the estimated maximum operating frequency can be over $100 \mathrm{MHz}$.

Table 3: Delay time estimated by Synopsys

\begin{tabular}{lllll}
\hline & 1st part & 2nd part & 3rd part & 4th part \\
\hline $\begin{array}{l}\text { Equivalent gate count } \\
\text { for combinational logics }\end{array}$ & 255.25 & 196 & 1076 & 275 \\
$\begin{array}{l}\text { Equivalent gate count } \\
\text { for registers }\end{array}$ & 132 & 140.25 & 232.5 & 100.75 \\
$\begin{array}{l}\text { Equivalent gate count } \\
\text { for interconnection }\end{array}$ & 0.77 & 0.15 & 1.81 & 0.46 \\
$\begin{array}{l}\text { Total count } \\
\text { Delay (ns) }\end{array}$ & 388.02 & 336.4 & 1310.3 & 376.2 \\
\hline
\end{tabular}

\section{Experimental results}

The proposed circuit has been realised by using $0.6 \mu \mathrm{m}$ standard cells [15] and fabricated in a $0.6 \mu \mathrm{m}$ SPDM
CMOS process. The photograph of this chip is shown in Fig. 6. A commercial DAC (AD9721) [16] is used to obtain the analogue output waveform. The measured performance of the DDFS is listed in Table 4. The transistor count used in this DDFS is 11721 and its core area is $0.95 \times 1.10 \mathrm{~mm}^{2}$ (excluding pads). The maximum operation frequency is $85 \mathrm{MHz}$. Its power consumption against the clock frequency is shown in Fig. 7. Fig. 8 shows the measured SNR against operating clock frequency. A typical spectrum of the DAC output at $10 \mathrm{MHz}$ is shown in Fig. 9 where the second harmonic distortion is $-85.84 \mathrm{dBc}$ and the phase noise is $114 \mathrm{dBc} / \mathrm{Hz}$ at an offset frequency of $10 \mathrm{kHz}$.

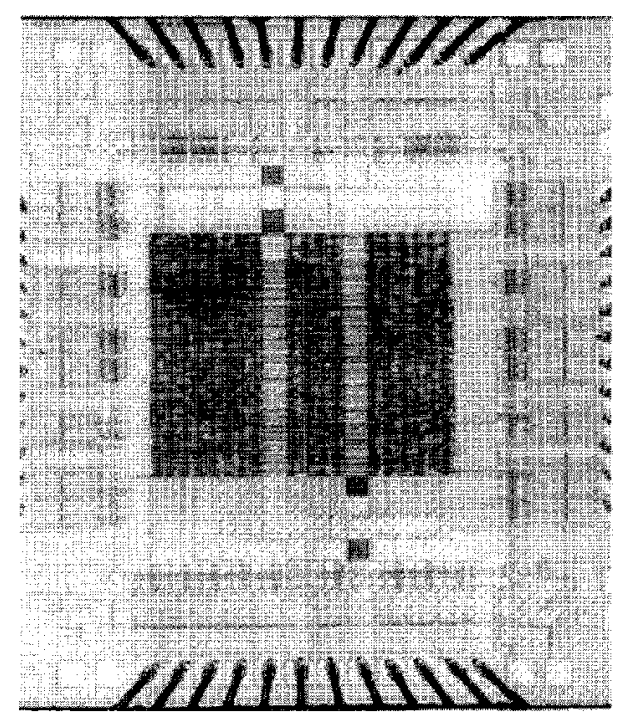

Fig.6 Photograph of proposed DDFS

Table 4: Measured performances of proposed DDFS

\begin{tabular}{ll}
\hline Frequency control word & 16 bits \\
Truncated phase control word & 14 bits \\
Output word length & 10 bits \\
Power supply & $5 \mathrm{~V}$ \\
Power dissipation & $80.7 \mathrm{~mW} @ 80 \mathrm{MHz}$ \\
Worst-case spurious & $-67.6 \mathrm{dBc} @ 10 \mathrm{MHz}$ \\
Phase noise & $-114 \mathrm{dBc} / \mathrm{Hz} @$ offset \\
& $10 \mathrm{kHz}$ from $10 \mathrm{MHz}$ \\
Chip Area & $0.95 \times 1.1 \mathrm{~mm}^{2}$ \\
& $($ excluding pads) \\
Package & DIP 48 -pin \\
Technology & $0.6 \mu \mathrm{m}$ SPDM CMOS \\
Design style & cell-based \\
Transistor count & 11721 \\
\hline
\end{tabular}




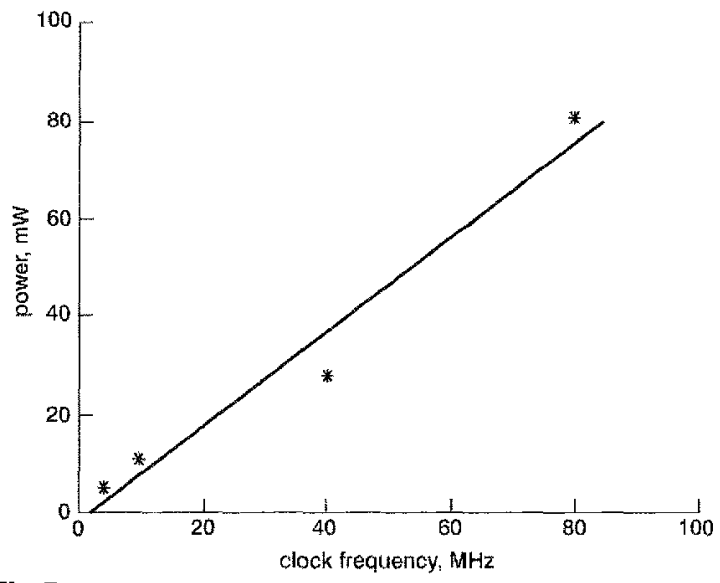

Fig.7 Measured power aguinst operuting clock frequency

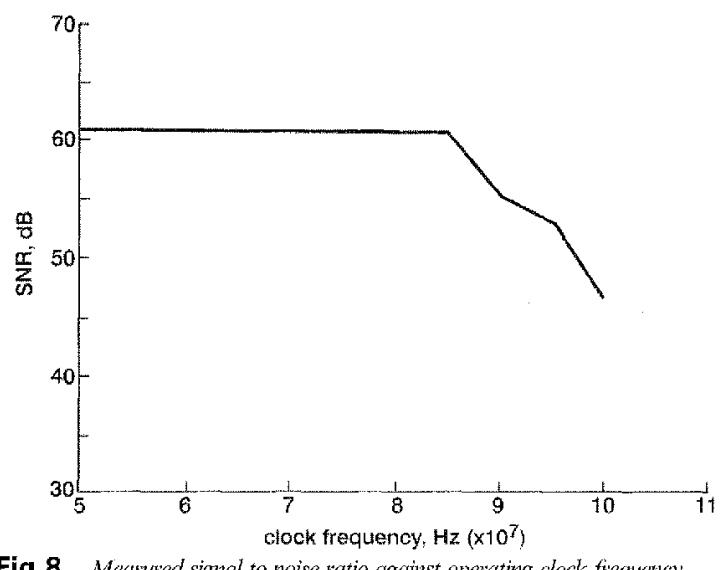

Fig. 8 Measured signal-to-noise ratio against operating clock fieguency

\section{Conclusions}

A pipeline DDFS using a new sine function decomposition method has been presented and fabricated in a $0.6 \mu \mathrm{m}$ SPDM CMOS process. Using the proposed decomposition method the pipeline DDFS without the sine ROM table has been realised. The cxperimental results clearly demonstrate the satisfactory performance of the proposed DDFS.

\section{Acknowledgment}

The authors thank the National Science Council for financial support and the Chip Implementation Center (CIC), National Science Council, Taiwan, Republic of China for the fabrication of the chip. This work was sponsored by NSC-88-2215-E-002-030.

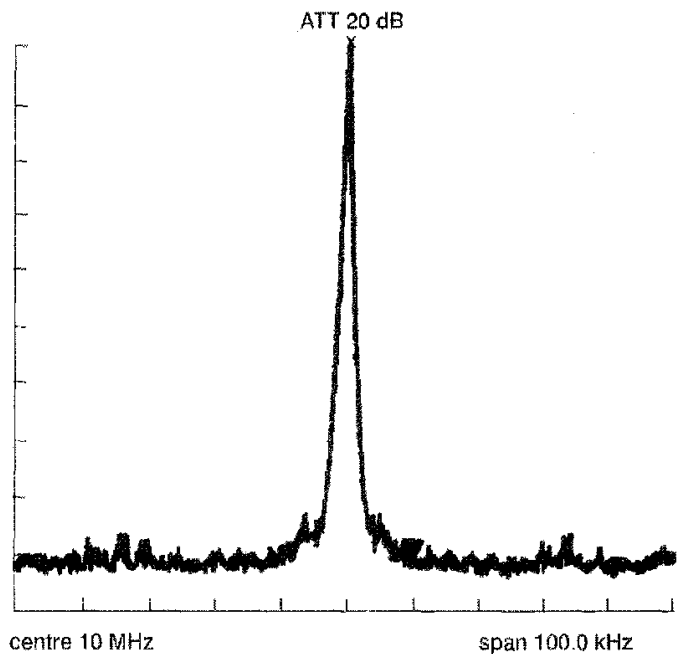

Fig.9 Measured spectrun of $10 \mathrm{MHz}$ output of profosed DDFS

\section{References}

1 MANASSEWITSCH, V.: 'Frequency synthesizers, theory and design' (Wiley, New York, 1979, 2nd edn.), p. 54

2 TIERNIYY, J., RADER, C.M., and GOLD, B.: 'A digital frequency synthesizer', IEEE Trans. Audio Electroacoust., March 1971, AU-19, pp. $48-56$

3 GORSKI-POPIEL, J.: "Frequency synthesis: techhiques and applications' (IEEJ Press, New York, 1975)

4 KROUPA. V.F. (Ed.): 'Direct digital frequency synthesizers' (IFEE Press, 1999)

5 SUNDERLAND, D., STRAUCI, R.. WHARFIELD, S., PETERSON, H., and COLE, C. 'CMOS/SOS frequency sunthesizer LSI circuit for spread spectrum communications', IEEE J. Solid-State Circuits, August 1984, SC-19, pp. 497-505

6 NICHOLAS, H.T., and SAMUELI, H.: "A $150 \mathrm{MHz}$ direct digital frequency synthesirer in $1.25 \mu \mathrm{m}$ CMOS with $-90 \mathrm{dBc}$ spurious perCormanco: IEEE J. Solid-State Circtits, December 1991, 26. pp. 19591969

7 TAN, I.K., and SAMUELI, H.: 'A $200 \mathrm{MH} /$ quadrature digital synthesizer/mixer in a $0.8 \mathrm{um}$ CMOS'. IEEE J. Solid-State Circuits, March 1995,30 , pp. 193-200

8 Qualcomm Q2334, techuical dala sheet, June 1991

9 VANKKA, J.: "Methods of mapping from phase to sine amplitude in direct digital synthesizer", IEEE Trans. Ultrason. Ferroelectr. Freq. Control, March 1997,44 , pp. $526-534$

10 CHRIN, W.A.: 'RNS-based enhancoments for direct digital frequency synthesis', IEEE Trans. Circuits Systens. II: Analog Digit. Signal Process. August 1994,42 , pp. 516-524

11 The Mathworks Inc.. MA, 1996

12 UYYA, M, and KANEKO, K.: 'А CMOS floating point muliplier'. IEEE ISSCC, digest of technical papers, February 1984, pp. 90-91

13 WESTE, N.H.E., and ESHIRAGHIAN. K. "Principles of CMOS VLSI design, A systems perspective' (Addison Wesley, 1993, 2nd edn.), p. 532

14 Synopsys Inc., Mountain Vicw, CA, 1995

15 Chip Implementation Center, National Seience Council, Hsinchu, Taiwan. Republic of China

16 Analog Devices, data converter reference manual, 1992 